\title{
Non-Violent Communication and Its Impact on Suicidal Ideations and Coping with Stress Styles
}

\author{
Heshmati-Molaie Nafise, Zandkarimi Ghazal* \\ Department of Psychology, Refah University, Tehran, Iran \\ Email: heshmati205@gmail.com, ^ghazalzandi@yahoo.com
}

How to cite this paper: Nafise, H.-M. and Ghazal, Z. (2018) Non-Violent Communication and Its Impact on Suicidal Ideations and Coping with Stress Styles. Journal of Behavioral and Brain Science, 8, 147-156. https://doi.org/10.4236/jbbs.2018.84009

Received: March 13, 2018

Accepted: April 20, 2018

Published: April 23, 2018

Copyright $\odot 2018$ by authors and Scientific Research Publishing Inc. This work is licensed under the Creative Commons Attribution International License (CC BY 4.0).

http://creativecommons.org/licenses/by/4.0/

(c) (i) Open Access

\begin{abstract}
One of the important dimensions of violence is suicide, or violence directed toward self that many people suffer from nowadays. In the meantime, adolescents and especially girls are more likely to be harmed. The purpose of this study was to evaluate the effect of group training with non-violent communication (NVC) method on suicidal ideations and coping with stress styles of adolescent girls. The target community in this research was availably selected from the charity centers of Tehran suburbs. In this study, 15 adolescent girls were in the experimental group and 15 in the control group. The criteria for entering the research were students aged 11 to 19 years and the vulnerable socio-economic income. Eight training sessions of NVC were presented for the experimental group in the pre-test and post-test intervals, while no intervention was performed for the control group. Data collection instruments were Beck's scale for suicidal ideation (BDI) and Endler \& Parker's coping inventory for stressful situations (CISS). After statistical analysis, the effectiveness of this training was investigated on dependent variables by multivariate analysis of covariance. Eventually, the results showed a significant difference between the experimental and control groups $(p<0.05)$. The finding of the study showed that NVC training can reduce suicidal ideations in adolescent girls who are socially and economically vulnerable. According to the findings, non-violent communication training has a higher effect on emotion-focused coping style compared to other stress coping styles.
\end{abstract}

\section{Keywords}

Adolescent Girls, Coping with Stress, Non-Violent Communication, Suicidal Ideation

\section{Introduction}

Adolescence can be considered as a period of life in which most of the social, 
psychological, cognitive and biological characteristics are changed. For this reason, people of this age are exposed to many mental and behavioral problems [1]. In the literature of social pathology, one of the obvious components of deviant behavior is poverty and economic weakness. Findings show that the culture of poverty exists in economically-harmed families, and vulnerable adolescents are more likely to be subjected to poor culture and beliefs than non-vulnerable ones [2] [3]. On the other hand, types of coping strategies can be one of the important prevention factors in teenager delinquency, and help them to adapt with changes and stressors [4].

Studies show that the adolescents with suicidal tendencies are poor in problem-solving and, therefore, the weakness in problem-solving skills is among the cognitive risk factors of suicide [5]. Endler and Parker suggested three types of coping styles: problem-focused, emotion-focused, and avoidance [6]. According to their definition, problem-focused coping occurs in situations where a person feels that the situation is controllable. As a result, he tries to solve and overcome the problem. Emotion-focused and avoidance coping occurs when a person feels that the situation is uncontrollable and thus, he tries to reduce or control the emotional distress posed by the situation. However, most psychological pressures arouse both types of coping, but when one is able to take advantage of the pressure, the problem-focused coping dominates. In contrast, when the only thing the individual can do is to impose or an emotional reaction to the problem, the emotion-focused or avoidant coping dominates. It seems that one of the ways people deal with stressful situations can affect suicidal ideation.

Of the most important predictors of suicidal behavior are violence, unsuccessful communication and suicidal ideation [7]. In addition, it seems that skill trainings such as non-violent communication that emphasizes in problem solving can be effective in reducing suicidal ideation. Non-violent communication (NVC) model is an interactive method that facilitates the exchange of information and peaceful resolution of disputes. It relies on shared human values and needs, and encourages people to refrain from language that leads to resistance or reduced self-esteem. Subsequently, it reinforces a communicational language that increases health, and emphasizes in the acceptance of responsibility in choosing and enhancing the quality of relationships [8].

Suicide statistics of Iran are high in the Middle East region; however, it should be remembered that Iran has entered a phase of social transition that is accompanied with transformation of values. Therefore, the need for further investigation of the causes and factors involved in this issue is necessary. Those who attempt to suicide are encountered with more stressful events, suffer from higher levels of psychological stress, and are awkward in use of problem-focused coping strategies. Regarding the sudden nature of suicide attempt and the risky age group (15 - 24 years), it has been shown that failure to reach mental maturity and lack of problem-solving skills, especially in this age range, can play the main role in suicide attempts. In other words, inefficient communication and emo- 
tional problems play a very important role in adolescences suicide [9]. In fact, those who commit suicide show a low performance in skills such as problem solving, emotional accompaniment, and overall family performance, wrong emotion-focused coping styles, and experienced more stressful events compared to the witness group [10].

A study determined the effectiveness of NVC intervention in the development of communication skills in male subjects with recorded crimes in treating drug abuse. Results showed that NVC training, in addition to positive impact on drug abuse treatment programs, was effective in dealing with communication styles caused by criminal behavior and provided supportive help in constructing social support networks [11]. In another study, it was found that non-violent behavior training reduces employee distress and anxiety. Also, it reduces the impact of environmental stressors due to increased ability of employees to control their feelings [12].

Intervention in suicide and stress coping styles in adolescents seems necessary, especially in vulnerable and poor groups of socio-economic income. Socially vulnerable individuals tolerate a higher level of helplessness in contrast with social pressures [13]. According to studies conducted, children and adolescents are more likely to be subjected to physical and sexual harm caused by familial violence, and this is higher in poor families [14]. In the meanwhile, adolescent girls are more vulnerable to cultural constraints and are more likely to commit suicide as a result of helplessness caused by violence and cultural restrictions [15]. Therefore, this study was conducted with the aim of teaching the NVC to reduce the suicidal ideations and correcting stress coping styles. The purpose was to find an answer to the question that whether NVC effects on the suicidal ideations of adolescent girls in the vulnerable groups of society and whether it could improve their stress coping style.

\section{Method}

\subsection{Study Population}

The present study method is a quasi-experimental study with pretest-posttest and control group which was performed in charity centers of Tehran suburbs for 8 weeks. Independent variable of this study was group training using NVC method and dependent variables were the level of suicidal ideation and three stress coping styles (problem-focused, emotion-focused, and avoidance). The statistical population was adolescent girls aged 11 to 18 of the vulnerable socio-economic income groups of Tehran suburbs. Thirty participants were selected availably and were randomly assigned to experimental and control groups. The observed power for the sample size (15 participants in each group) was in middle level, as the high power is depend on the sample more than 25 participants in each group [16]. The criteria for entering the study were age and gender, being a student and being a member of lower socio-economic class. It should be noted that all ethical criteria were observed. Firstly, we collected the consent of parents, be- 
cause the participants were under the age of 21 . Then the procedure of research was clarified to the participants and all of the security items such as confidentiality of information and protection from harms were regarded. Moreover, all participants had the right of withdraw from the study in any period of the research.

\subsection{Instruments}

Beck's Scale for Suicidal Ideation (BSSI) was developed to detect and measure the intensity of attitudes and planning for committing suicide [17]. This scale contains 19 questions and its total score is from 0 to 38 . According to study the reliability of this scale with Cronbach's alpha coefficient has been reported to be [18] 0.89 in adults. Moreover, the reliability of BDI was reported as 0.94 by Cronbach's alpha for 140 girl students (11 - 18 years) of Tehran suburban. In addition, convergent validity with each of the DASS-21 subscales was between 0.63 and 0.73 , and divergent validity with a positive mental health scale (PMH) was $-0.71[19]$.

Coping Inventory for Stressful Situations (CISS) consists of 48 items that examines the problem-focused, emotion-focused and avoidance coping styles [6]. The dominant coping style of each individual is determined by their higher score in each style. Endler and Parker reported using Cronbach's alpha, which is 0.82 to 0.92 of internal consistency for subscales. In this research, the test-retest correlation coefficient for the sub-scales rangs between 0.77 and 0.90 .

Non-violent Communication (NVC) training protocol, the main contents of each session was as follows (Table 1) [8].

\subsection{Procedure}

A pre-test was administered to experimental and control groups before holding

Table 1. Headings of each session.

\begin{tabular}{|c|c|}
\hline Session & Headings \\
\hline 1 & $\begin{array}{l}\text { Pre-test before the session. Getting familiar with the participants; getting familiar with } \\
\text { violence and its types; pointing out the uses of NVC in everyday life; simulation of } \\
\text { violent communication. }\end{array}$ \\
\hline 2 & $\begin{array}{l}\text { Discussing the need for thinking and accuracy; a brief discussion about non-violent } \\
\text { communication and its stages; practical exercise. }\end{array}$ \\
\hline 3 & First stage of NVC (observance without judging); practical exercise. \\
\hline 4 & $\begin{array}{l}\text { Kindness-blocking communication: Ethical Judgment and Comparison; practice and } \\
\text { simulation of unsuccessful conversations. }\end{array}$ \\
\hline 5 & Second stage of NVC (express feelings); practical exercise. \\
\hline 6 & $\begin{array}{l}\text { Accept responsibility for your feelings and needs; understanding and expressing } \\
\text { needs and demand for enriching our lives; practical exercise. }\end{array}$ \\
\hline 7 & $\begin{array}{l}\text { Training sympathy and kind communication with oneself and others; practical } \\
\text { exercise. }\end{array}$ \\
\hline 8 & $\begin{array}{l}\text { Reviewing the steps of NVC method and double play; post-test was administered at } \\
\text { the end of the session. }\end{array}$ \\
\hline
\end{tabular}


the 8 training sessions using NVC method. Subsequently, the post-test was performed after the last session. This measurement was performed individually using scales and under the supervision of the examiner, as to better control possible ambiguities. The demographic data of the participants age represented that 4 percent of them were in the age of 11,9 percent in 12, 12 percent in 13, 32 percent in 14, 13 percent in 15,10 percent in 16,14 percent in 17, and 6 percent were in the age of 18 . So, the frequency table of the sample group showed that participants aged 14 were in higher frequency and participants aged 11 and 18 had lower frequency in this study. The data obtained from the research was organized and SPSS-24 software was used for data analysis.

\section{Results}

The descriptive results of the experimental and control groups are shown in the table with respect to dependent variables.

Table 2 shows the average and standard deviation of the research variables. According to the table, the average suicidal ideation of the experimental group decreased in the post-test. Moreover, the emotion-focused style decreased in the post-test, although multivariate analysis of covariance can be decisive in determining the significance of the differences. All the assumptions of this test were examined. Skewness and kurtosis scores of variables were evaluated in pre-test and post-test. All of which were under 2. Since our sample constituted less than 30 participants, the use of the Kolmogorov-Smirnov test was inappropriate and instead skewness and kurtosis scores of the data were considered. All of skewness and kurtosis were below 1.50, indicating that their distribution was normal [16]. In addition, the correlation between dependent variables was in middle level between 0.35 and 0.55 , which indicated that they are not overlapped $(p<$ $0.05)$.

One of the important hypotheses in multivariate analysis of covariance is the homogeneity of covariance matrices in experimental and control groups. For this purpose, Box's $\mathrm{M}$ test was applied and the results of $\mathrm{F}_{(1.28)}=0.76$ and $(p<$ $0.59)$ represented the homogeneity of dependent variables covariance matrix. In addition, Levene's test was used to examine the homogeneity of the dependent variables variance matrix. The results of which were $\mathrm{F}_{(1.28)}=0.35,0.79,0.02$

Table 2. Descriptive statistics of suicidal ideations and stress coping styles.

\begin{tabular}{cccccccccc}
\hline & \multicolumn{3}{c}{ Examination Group } & \multicolumn{3}{c}{ Control group } \\
\cline { 2 - 9 } Variable & \multicolumn{2}{c}{ Pre test } & \multicolumn{2}{c}{ Post test } & \multicolumn{2}{c}{ Pre test } & \multicolumn{2}{c}{ Post test } \\
\cline { 2 - 10 } & $\mathrm{M}$ & SD & M & SD & M & SD & M & SD \\
\hline Suicidal Ideations & 8.93 & 9.22 & 4.70 & 7.22 & 9.13 & 8.03 & 9.02 & 8.08 \\
Problem-Focused Coping & 34.53 & 10.45 & 33.93 & 11.18 & 31.00 & 9.59 & 30.80 & 9.70 \\
Emotion-Focused Coping & 29.33 & 9.44 & 28.53 & 9.48 & 29.40 & 10.24 & 29.47 & 9.79 \\
Avoidant Coping & 31.90 & 10.25 & 31.07 & 11.65 & 25.60 & 7.95 & 25.53 & 7.90 \\
\hline
\end{tabular}


and 0.42 for the variables of suicidal ideation, problem-focused, emotion-focused and avoidance coping respectively. The significance level was between 0.38 and 0.87 , indicating the homogeneity of the variance matrix in dependent variables. The last hypothesis was to investigate the independence of errors, which should be examined by the residual plots. According to residual plots, the normality hypothesis was observed.

According to Table 3 and with regard to the significant values that are above 0.05 , the significant difference was observed of suicidal ideations and coping styles between the experimental and control groups.

\section{Discussion}

The purpose of the present study was to evaluate the effectiveness of NVC training on adolescent girls' suicidal ideations and coping with stress styles. Moreover, we aimed to extend the knowledge boundaries of non-violence communication training in adolescence. The researchers found interesting findings after performing the intervention. The main findings are the following.

Firstly, NVC training significantly reduced the suicidal ideations of vulnerable adolescent girls. The findings of this study will help clarify the positive effect of training the communication skills on suicidal ideations and attempts. However, using NVC method to increase interpersonal skills in this study is questionable itself that will be explained in what follows. The effect of NVC on reducing suicidal ideation was also consistent with the findings of other researchers. Walker and Ziebeck also found that non-violent communication education helps to increase self-control feeling [12]. At the same time, Flory and Colleagues also showed that lack of healthy communication skills and inability in problem solving increases suicide attempts among adolescents. Therefore, communication skills training represent a good effect on reducing suicidal ideation [9]. Given that, we know emotional-communication problems play a very important role in suicidal attempts in adolescents. Regarding to this finding, the necessity of working on this group and confirming the hypothesis in the juvenile group can also be explained. It can be said that adolescence is a transition period from childhood to adult responsibility, and the adolescents try to have autonomy and independence. For this purpose, they must be able to form a separate social and familial identity through effective communication with their parents. The

Table 3. Multivariate covariance analysis of suicidal ideation and coping styles with pre-test effect control.

\begin{tabular}{ccccccc}
\hline \multirow{2}{*}{ Factor } & Dependent variable & Total squared & Df. & Mean of squares & F & Sig. \\
\cline { 3 - 5 } & Suicidal Ideations & 77.09 & 1 & 77.09 & 6.13 & 0.02 \\
\multirow{2}{*}{ Group } & Problem-Focused Coping & 1479.76 & 1 & 1479.76 & 19.49 & 0.01 \\
& Emotion-Focused Coping & 3947.59 & 1 & 3947.59 & 28.49 & 0.01 \\
& Avoidant Coping & 2335.70 & 1 & 2335.70 & 21.00 & 0.01 \\
\hline
\end{tabular}


increasing efforts of the adolescents to achieve autonomy can lead to increased conflicts with parents and the creation of negative feelings. These familial conflicts can be due to weak interpersonal skills and insufficient ability to control anger. At the same time, if the socioeconomic situation in developmental period is accompanied by poverty, prejudice and violence, transition from adolescence to adulthood would be stressful. In addition, gaining independent identity can turn into a nightmare that one may decide to put an end to his life to get rid of it. The findings of this study showed that training NVC in the adolescent group from vulnerable social class resulted in the reduction of self-directed aggression and thus of suicidal ideation and beliefs. This suggests that, regardless of the inappropriate socioeconomic environment, it is possible to correct and control ideation and beliefs, and non-violent communication has been very influential in this regard.

Secondly, group training through NVC method significantly effects on stress coping strategies (problem-focused, emotion-focused and avoidance styles) of adolescent girls. Especially regarding the emotion-focused coping style, this difference was more tangible. According to the findings, it seems that non-violent communication training can cause people to move from emotion-focused and aggressive coping strategies such as self-harm and harming others, verbal aggression and behavioral emotions towards effective and constructive emotional coping. The most prominent of healthful emotional coping styles are breathing techniques, self-relaxation, change of attention, etc. The results of this finding are consistent with other studies. For example, Ghadampour \& Colleagues, also concluded that effective communication training can reduce the sense of helplessness in dealing with stress [13]. Furthermore, Wacker and Dziobek, found that staff distress and anxiety were reduced, and the impact of environmental stressors also decreased due to control of thoughts and emotions by employees [12]. In another study aimed at determining the effectiveness of behavioral-communication intervention in the development of coping and communication skills, there was a significant increase in the level of the empathy of the participants [11].

Finally, after the significant impact of NVC on emotion-focused coping, problem-focused coping was also significantly influenced. In fact, the results represented that NVC training increased the abilities of problem-solving, concentration on situation and peaceful conflict resolution in adolescents. Stress coping style has a significant correlation with suicidal ideation. According to the findings of Harms, those who have a poor problem solving ability are more likely to suicide as a strategy to get rid of the problems [10]. Therefore, given the educational, mental and psychological preoccupations of adolescent students and environmental stresses, suitable strategies for coping with problem-focused stress can activate appropriate strategies in their minds. Consistent with above results, the positive effects of NVC training were also observed in this study. Despite the fact that this training is more necessary for poor society and the 
weak population due to their particular problems and less access to educational programs, less attention is paid to such training in these particular environments in Iran.

The present study has powerful points such as training, intervention, and evaluation of effectiveness level on the participants of the sample group. Moreover, the findings of this study revealed significant information on suicide and stress coping styles of adolescent girls from poor socio-economic income. However, this study, like any other studies, has some limitations that should be considered in subsequent studies. Firstly, individual differences such as intelligence and environmental factors such as happy or unpleasant events during the training sessions might be influence the participants' mood throughout the session while filling out the questionnaires. However, these components were not considered as affecting suicidal ideation. As a result, when the participants were responding to the questionnaire items, it was observed that some participants, responded better than others and were more focused despite the same level of training. Secondly, it was not possible to hold a follow up course in this study. Due to this limitation, the effectiveness of the intervention was not investigated over time. Therefore, it is suggested that the factors of intelligence and environmental stress be considered for each participant in future studies. It is also necessary to consider the follow-up phase in subsequent studies. Finally, non-violent communication training should be taught to children of both genders in environments with low socio-economic income to protect them from suicide due to familial-environmental reasons.

\section{Conclusion}

The aim of this study was to investigate the effect of non-violent communication on the suicidal ideation and stress coping styles of female adolescent students. The main purpose of the study was to develop the knowledge of this field and to demonstrate the effect of non-violence communication training so that this training is used for adolescent students. The results of this study showed that group training through non-violent communication method showed a significant effect on the reduction of suicidal ideation and ability to use of suitable stress coping styles in female adolescent students. This effect on stress coping styles was first noticeable in the emotion-focused style and then in the problem-focused style.

\section{Acknowledgements}

Funding for this work was provided by Refah University. The authors thank the participating female adolescent students who assisted in interpretation of the results. The authors declare that they have no competing or potential conflicts of interest. All ethical considerations (e.g. participants' informed consent, security, safety and parental permission for they were under 21 years old) were regarded. The second author takes full responsibility for the integrity of the data and the 
accuracy of the data analyses.

\section{References}

[1] Lerner, R.M. and Galambos, N.L. (1998) Adolescent Development: Challenges and Opportunities for Research, Programs, and Policies. Annual Review of Psychology, 49, 413-446. https://doi.org/10.1146/annurev.psych.49.1.413

[2] United Nations (2015) The World's Women 2015: Trends and Statistics. United Nations, Department of Economic and Social Affairs, Statistics Division, New York.

[3] Elliott, I. (2016) Poverty and Mental Health: A Review to Inform the Joseph Rowntree Foundation's Anti-Poverty Strategy. Mental Health Foundation, London.

[4] Markova, S. and Nikitskaya, E. (2017) Coping Strategies of Adolescents with Deviant Behavior. International Journal of Adolescence and Youth, 22, 36-46. https://doi.org/10.1080/02673843.2013.868363

[5] Zong, S. (2015) A Study on Adolescent Suicide Ideation in South Korea. Journal of Social and Behavioral Sciences, 174, 1949-1956.

https://doi.org/10.1016/j.sbspro.2015.01.860

[6] Endler, N.S. and Parker, J.D. (1999) Coping Inventory for Stressful Situations (CISS): Manual. 2nd Edition, Multi-Health Systems, Toronto.

[7] Glenn, C.R. and Nock, M.K. (2014) Improving the Prediction of Suicidal Behavior in Youth. International Journal of Behavior Consultation and Therapy, 9, 7-10.

[8] Rozenburg, M.B. (1983) Non-Violence Communication: A Language of Life. Lucy Leu, Wisconsin.

[9] Flouri, E., Narayanan, M.A. and Nærde, A. (2018) Stressful Life Events and Depressive Symptoms in Mothers and Fathers of Young Children. Journal of Affective Disorders, 230, 22-27. https://doi.org/10.1016/j.jad.2017.12.098

[10] Harms, P.D., Krasikova, D.V., Vanhove, A.J., Herian, M.N. and Lester, P.B. (2013) Stress and Emotional Well-Being in Military Organizations. Bulletin of Nebraska Public Policy Center, 11, 103-132. https://doi.org/10.1108/S1479-3555(2013)0000011008

[11] Marlow, E., Nyamathi, A.T., Grajeda, W., Bailey, N., Wwber, A. and Younger, J. (2011) Nonviolent Communication Training and Empathy in Male Parolees. Journal of Correctional Health Care, 18, 8-19. https://doi.org/10.1177/1078345811420979

[12] Wacker, R. and Dziobek, I. (2016) Preventing Empathic Distress and Social Stressors at Work through Nonviolent Communication Training: A Field Study with Health Professionals. Journal of Occupational Health Psychology, 23, 141-150. https://doi.org/10.1037/ocp0000058

[13] Ghadampour, F., Shafiei, M. and Heidarirad, H. (2017) Relationships among Cyberbullying, Psychological Vulnerability and Suicidal Thoughts in Female and Male Students. Journal of Research in Psychological Health, 11, 28-40. https://doi.org/10.29252/rph.11.3.28

[14] Dupere, V., Leventhal, T. and Lacourse, E. (2009) Neighborhood Poverty and Suicidal Thoughts and Attempts in Late Adolescence. Journal of Psychological Medication, 39, 1295-306. https://doi.org/10.1017/S003329170800456X

[15] Alwan-Baiee, H., Fadhil, Z., Salman, J. and Sheehan, S. (2017) Prevalence of Suicide Ideation among Iraqi Secondary School Students in Babylon Province during the Academy Year 2016-2017. Medical Journal of Babylon, 14, 577-584.

[16] Gamst, G., Meyers, L.S. and Guarino, A.J. (2008) Analysis of Variance Designs. 
Cambridge University Press, New York.

https://doi.org/10.1017/CBO9780511801648

[17] Beck, A.T., Kovasac, M. and Weissman, A. (1979) Assessment of Suicidal Intention: The Scale for Suicide Ideation (SSI). Journal of Consulting Clinical Psychology, 47, 343-352. https://doi.org/10.1037/0022-006X.47.2.343

[18] Anisi, J., Fathi-Ashtiani, A., Salimi, H. and Ahmmadi, K.H. (2005) Evaluation of Validity and Reliability of Beck Suicide Scale Ideation (BSSI) in Soldiers. Journal of Military and Medication, 7, 33-37.

[19] Heshmati-Moulai, N., Jahangiri, F. and Zandkarimi, G. (2017) Investigation the Psychometric Properties of Beck Suicidal Scale Ideation in Girl Adolescents. $3^{\text {th }}$ International Conference on Recent Innovations in Psychology, Kharazmi University, Tehran, Iran. 